\title{
Dilemmas in autoimmune pancreatitis. Surgical resection or not?
}

\author{
Hoffmanova $\mathrm{I}^{1}$, Gurlich $\mathrm{R}^{2}$, Janik $\mathrm{V}^{3}$, Szabo $\mathrm{A}^{4}$, Vernerova $\mathrm{Z}^{4}$ \\ Second Department of Internal Medicine, Third Faculty of Medicine, Charles University \\ in Prague, Czech Republic. iva.hoffmanova@fnkv.cz
}

\begin{abstract}
Surgical treatment is not commonly recommended in the management of autoimmune pancreatitis. The article describes a dilemma in diagnostics and treatment of a 68-year old man with the mass in the head of the pancreas that mimicked pancreatic cancer and that was diagnosed as a type 1 autoimmune pancreatitis (IgG4related pancreatitis) after a surgical resection. Diagnosis of the autoimmune pancreatitis is a real clinical challenge, as in the current diagnostic criteria exists some degree of overlap in the findings between autoimmune pancreatitis and pancreatic cancer (indicated by the similarity in radiologic findings, elevation of IgG4, sampling errors in pancreatic biopsy, and the possibility of synchronous autoimmune pancreatitis and pancreatic cancer). Despite the generally accepted corticosteroids as the primary treatment modality in autoimmune pancreatitis, we believe that surgical resection remains necessary in a specific subgroup of patients with autoimmune pancreatitis (Fig. 4, Ref. 37). Text in PDF www.elis.sk.

KEY WORDS: autoimmune pancreatitis, IgG4-related pancreatitis, obstructive jaundice, resection of pancreas.
\end{abstract}

List of abbreviation: AIP - autoimmune pancreatitis, ERCP endoscopic retrograde cholangiopancreatography, IgG4 - imunoglobulin G subtype 4, IgG4-RD - IgG4-related disease

\section{Introduction}

The term "autoimmune pancreatitis" (AIP) was first proposed by Yoshida and colleagues in 1995(1). AIP is a rare disorder, with a reported overall prevalence known only in Japan, of approximately $2.2 / 100000$ (2). The peak age of onset is in the seventh decade, with $95 \%$ of patients older than 45 years $(2,3)$. AIP is at least twice common in men as in women (in 4) and represents 2-10\% of the patients with chronic pancreatitis (2). AIP is a unique form of chronic pancreatitis, which could be classified into two distinct subtypes, type 1 and type 2 (4-6).

Type 1 autoimmune pancreatitis (type 1 AIP) is also known as IgG4-related pancreatitis or lymphoplasmocytic sclerosing pancreatitis $(7,8)$. It is considered to be a part of a systemic disease named IgG4-related disease (IgG4-RD) and associated with elevated levels of imunoglobulin G subtype 4 (IgG4) producing plasma cells and serum IgG4 elevation (>140 mg/dl) (7, 9). IgG4-RD has been recognized as a novel clinical entity with

\footnotetext{
${ }^{1}$ Second Department of Internal Medicine, Third Faculty of Medicine, Charles University in Prague, ${ }^{2}$ Department of General Surgery, Third Faculty of Medicine, Charles University in Prague, ${ }^{3}$ Department of Radiodiagnostics, Third Faculty of Medicine, Charles University in Prague, and ${ }^{4}$ Department of Pathology, Third Faculty of Medicine, Charles University in Prague, Czech Republic
}

Address for correspondence: I. Hoffmanova, MD, PhD, 2nd Department of Internal Medicine, Third Faculty of Medicine, Charles University in Prague, Ruska 87, CZ-100 00 Prague 10, Czech Republic Phone: +420.267162703 , Fax: +420.267162710 multiorgan involvement and an abnormal immunological process of unknown origin; involved organs showing diffuse or focal enlargement and mass-forming or nodular/thickened lesions due to the prominent infiltration of lymphocytes and IgG4-positive plasma cells associated with fibrosis (7). IgG4-RD has been found to affect the pancreas, bile duct tree, liver, gastrointestinal tract, lacrimal glands, salivary glands, central nervous system, thyroid, lungs, kidneys, prostate, retroperitoneum, mediastinum, arteries, lymph nodes, skin, and breast $(4,10)$. The diagnosis of type 1 AIP is based on histopathologic features characterized by a diffuse lymphoplasmacytic infiltrate with abundant infiltration of IgG4+ plasmocytes $>10$ cells per high power field and fibrosis. The fibrosis is invariably organized in a storiform pattern. The inflammatory cells tend to aggregate around ducts, but the periductal infiltrate is seldom as prominent as seen in type 2 AIP and ductal epithelium is preserved. The inflammatory infiltrate also extends into peripancreatic adipose tissue. Obliterative flebitis is readily identified (11).

Type 2 autoimmune pancreatitis (type 2 AIP) is a specific pancreatic disorder, which is not a part of the IgG4-RD spectrum (8) and has different histologic pattern (a dense periductal collar of lymfoplasmacytic inflammation, accompanied by neutrophilic microabscesses within the lumen of the duct, the so called granulocytic epithelial lesion, which often causes destruction and obliteration of the pancreatic duct). The type 2 AIP lacks both elevated levels of serum IgG4 and IgG4-positive plasma cells (6).

The most common presenting symptom of the AIP is obstructive jaundice (up to $75 \%$ of cases) secondary to entrapment of the intrapancreatic bile duct by the inflammatory process and aggravated by the IgG4-associated cholangoitis. This occurs in approximately $75 \%$ of patients with the type 1 AIP and $50 \%$ of patients with the type 2 AIP. However, patients with the type 2 
463-467

AIP present more commonly with acute pancreatitis (34\%) and abdominal pain (68\%) than patients with the type 1 AIP (12).

A variety of diagnostic scoring systems for the AIP have been advocated around the world. In the United States, Chari and colleagues (13) introduced the mnemonic HISORt criteria based on di-

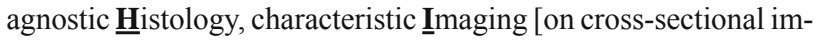
aging with contrast-enhanced computed tomography or magnetic resonance imaging is pancreas diffusely enlarged, with featureless borders and/or loss of lobular architecture, or "sausage-shaped"

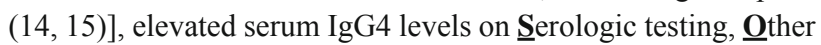
organ involvement (in type 1 AIP), and $\underline{\mathbf{R}}$ esponse to glucocorticoid therapy. It was one of the most commonly used diagnostic criteria in the United States.

In 2011, an international panel of experts developed International Consensus Diagnostic Criteria (ICDC) for the AIP, which focused on the distinction between type 1 and type 2 AIP (6). These criteria are based on the clinical profile of AIP, including characteristic histology, and imaging, serum IgG4 levels, extrapancreatic manifestations, and response to steroid treatment. For each criterion, there are two levels of evidence: typical or highly suggestive evidence (level 1), and indeterminate/suggestive evidence (level 2 ). With this stratification, the type 1 AIP can be confirmed with a variety of combinations of level 1 and level 2 evidence. However, definitive diagnosis of the type 2 AIP requires histology $(6,11)$.

Histology is the "gold standard" for the diagnosis of the AIP. Tissue acquisition for histological diagnosis may be obtained by endoscopic ultrasound (EUS) with core biopsy $(4,16)$. Fine needle aspiration biopsy, which typically obtain only aspirate for cytology is not recommended for the diagnosis. Core biopsy needle preserves tissue architecture, allowing immunostaining and examination to diagnose AIP (17). The study from Denmark has recently demonstrated that laparoscopic or percutaneous ultrasound-guided core needle biopsy had the highest sensitivity for diagnosis of AIP in comparison to endoscopic ultrasound-guided core needle biopsy (18).
The AIP is referred to be very responsive to steroid therapy, therefore making therapy a component of the diagnostic criteria $(4,16,19-21)$.One commonly used regimen includes treatment with $40 \mathrm{mg}$ of prednisone for four weeks, followed by a taper by $5 \mathrm{mg}$ each week for a total of an11-week course (11). Patients, who relapse, are treated with a second course of corticosteroids (19). Given the relatively high relapse rates, some centres routinely continue maintenance corticosteroid therapy for up to three years (21). Further, the steroid-sparing immunomodulator, azathioprine, can be used to maintain remission after the first or second relapse (19). In patients refractory to steroids, azathioprine, mycophenolate mofetil, cyclophosphamide, and rituximab have all been tried in addition to, or instead of, steroid therapy $(16,20)$.

\section{Material and methods}

68-year-old man presented with a history of weight loss about seven kilograms in seven weeks and obstuctive jaundice. A physical examination was normal with the exception of jaundice; there was no evidence of ascites, lymphadenopathy or hepatosplenomegaly. Routine laboratory analysis revealed typical sign of obstuctive icterus (total bilirubin $97.1 \mathrm{umol} / \mathrm{l}$, alkaline phosphatase 2.70 ukat/l, $\gamma$-glutamyl transpeptidase 5.14 ukat/1, aspartate aminotransferase $2.35 \mathrm{ukat} / \mathrm{l}$, alanine aminotransferase $3.90 \mathrm{ukat} / \mathrm{l}$ ), and borderline levels of serum amylase (1.95 ukat/), serum lipase (1.90 ukat/l). Level of C-reactive protein $(0.5 \mathrm{mg} / \mathrm{l})$ and tumor marker CA 19-9 (10.0 kU/l) was normal. Complete blood count was normal. Abdominal ultrasound examination reported hypoechoic $50 \mathrm{~mm}$ large lesion in head of pancreas; gallblader was normal without lithiasis. Abdominal computed tomography (Fig 1a,b) showed enlargement of the head of the pancreas $(52 \times 38 \times 31$ $\mathrm{mm}$ ) in comparison to the atrofic pancreatic body and tail, slightly dilatated pancreatic duct $(3 \mathrm{~mm})$ with concomitant common bile duct dilatation $(11 \mathrm{~mm})$, and slight intrahepatic bile duct dilata-
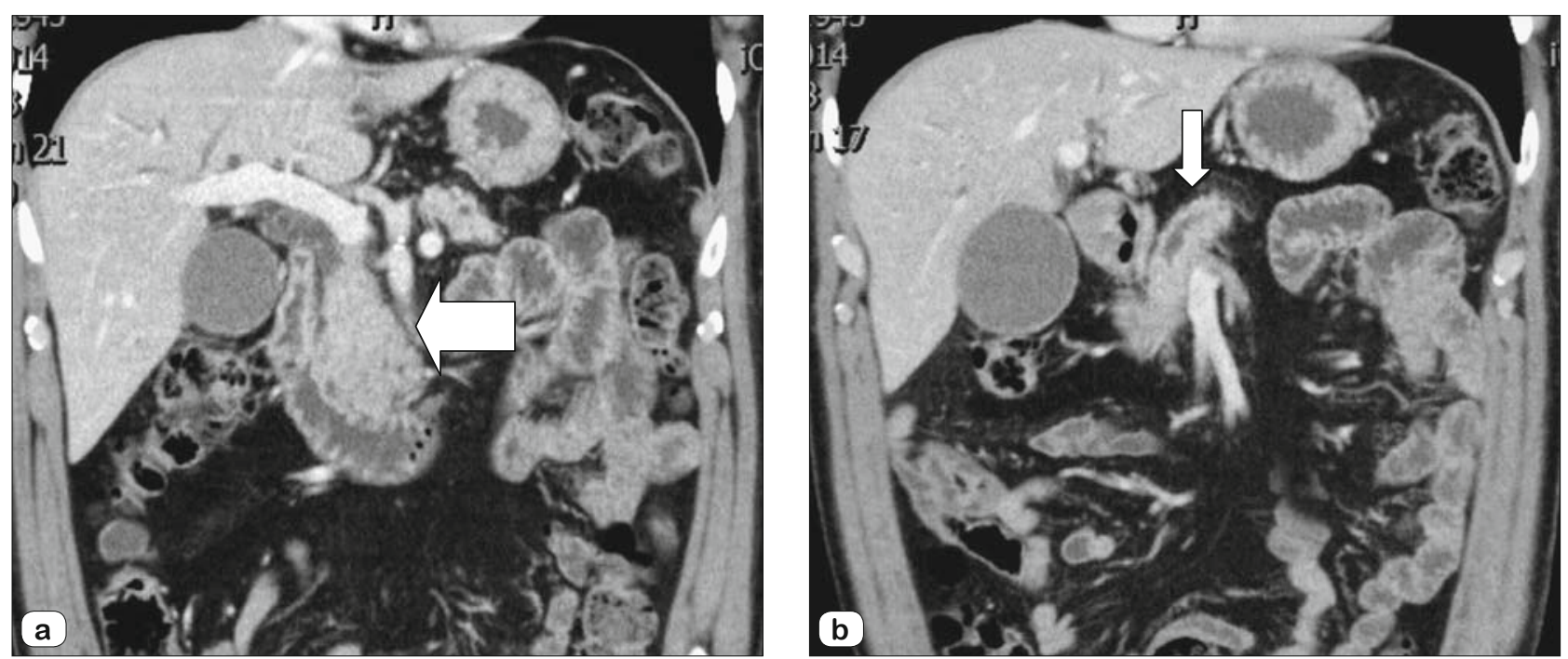

Fig. 1a, b. Coronal reconstruction contrast-enhanced CT images showed (a) enlargement of the pancreatic head (thick arrow) in comparison (b) to the atrofic pancreatic body with dilatated pancreatic duct (thin arrow). 


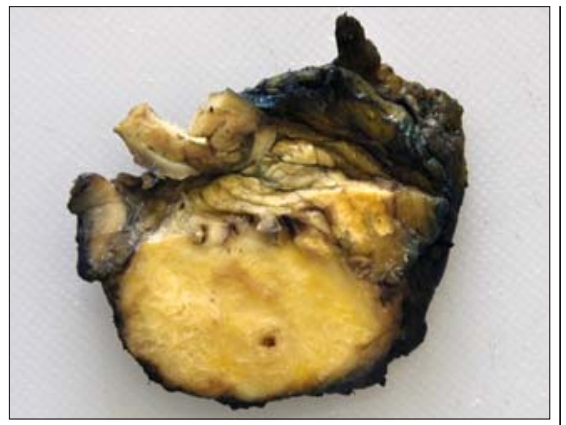

Fig. 2. A formalin-fixed lamelle of the resection specimen. A firm, fibrotic, poorly defined mass lesion was found in the pancreatic head, mimicking the appearance of pancreatic adenocarcinoma.

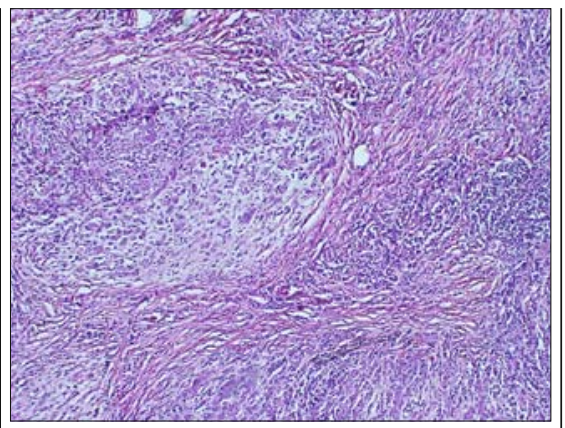

Fig. 3. Low-power view showed an extensive storiform fibrosis accompanied by a dense lymphoplasmocytic infiltrate. In spite of the destruction of the acini the lobular architecture of the pancreas was preserved, (HE, original magnification $\times 40$ ).

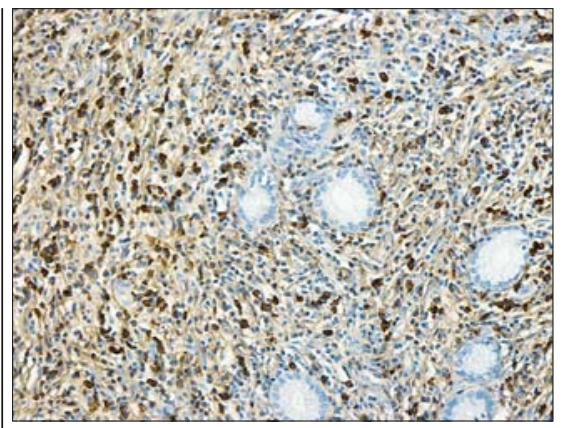

Fig. 4. The IgG4-immunostaining highlighted an increased number of IgG4-positive plasma cells in the markedly fibrotic interstitial stromal tissue. Of the original pancreatic tissue, only focally proliferating ductules are preserved, (original magnification $\times \mathbf{2 0 0}$ ). tion. Endoscopic retrograde cholangiopancreatography (ERCP) revealed edema in D2 part of duodenum and extreme rigidity of papilla of Vater. Because of the rigidity, biliary plastic stent was unable to place even by very experienced endoscopist.

Patient refused a pancreatic biopsy. In case of increasing obstructive jaundice without a possibility to insert biliary stent via ERCP, we considered steroid treatment for 2-4 week as a therapeutic modality that could increase risk of acute cholangitis. Alternative percutaneous biliary drainage in combination with steroid trial would share the same risk.

The patient was referred for surgical management. In keeping with preoperative examination, the surgical exploration demonstrated firm mass in the pancreatic head extending into the body and tail of pancreas. The patient underwent a standard pylorus preserving total pancreatectomy with splenectomy and standard lymphadenectomy. In reconstruction phase retrocolic reconstruction with a single limb for all anastomoses (hepaticojejunostomy and duodenojejunostomy) was performed.

Gross pathological finding showed a not well circumscribed firm grey to tan mass $(45 \times 35 \times 65 \mathrm{~mm})$ in the pancreatic head (Fig. 2). Microscopic evaluation revealed dense periductal lymphoplasmocytic infiltration, storiform intralobular fibrosis with secondary atrophy of exocrine acinar component (Fig. 3) and obliterative phlebitis. The IgG4 immunohistochemistry showed dense infiltration of IgG4-positive plasma cells (70 cells per HPF) (Fig. 4). Fibrosis and scaring involved also distal part of common bile duct leading to its obstruction.

Subsequently we obtained the result of IgG4 in serum. IgG4 level of $3.52 \mathrm{~g} / \mathrm{l}(352 \mathrm{mg} / \mathrm{dl})$ well corresponded with diagnosis of type 1 autoimmune pancreatitis.

\section{Discussion}

The clinical presentation of autoimmune pancreatitis can mimic difficult-to-treat disorders such as pancreatic cancer (16). Autoimmune pancreatitis most often presents with obstructive jaundice, weight loss, abdominal pain and new onset of diabetes mellitus $(16,22)$. This presentation is similar to that of pancreatic cancer. In $20-40 \%$ of cases of the AIP, a focal pancreatic mass is found, which makes the distinction from pancreatic cancer rather difficult $(16,23)$.

Above mentioned diagnostic criteria for AIP should be applied with caution as there is some degree of overlap in the findings between the AIP and pancreatic cancer indicated by the similarity in radiologic findings, elevation of IgG4, sampling errors in pancreatic biopsy, and the possibility of synchronous AIP and pancreatic cancer (24).

Serum IgG4 levels alone are unsuitable for distinguishing AIP from pancreatic cancer; serum IgG4 level > $140 \mathrm{mg} / \mathrm{dl}$ has a sensitivity of $76 \%$ and specificity of $93 \%$ in diagnosing of AIP (9). Notably, $5 \%$ of healthy persons and approximately $10 \%$ of the patients with pancreatic cancer have slightly (about 2-fold) elevated IgG4 $(9,25,26)$. Increasing the cutoff to $280 \mathrm{mg} / \mathrm{dl}$ decreases sensitivity, but improves specificity to $99 \%$ in diagnosis of AIP (16).

Diagnostic accuracy of serum biomarker CA 19-9 level is limited as well, as $9 \%$ of the patients with AIP have elevated CA 19-9. However, patients with pancreatic cancer are more likely to have elevated CA $19-9$ of $>100 \mathrm{U} / \mathrm{ml}$ than AIP patients (71\% vs 9\%) (9).

Bioptic sample errors could be possible because of the patchy distribution of the characteristic histological findings in AIP (17).

Diagnostic criteria for AIP include response to steroid therapy. However, steroid trials should be performed only after a negative workup for pancreatobiliary cancer (24). It had been shown that radiological improvement of the pancreas on cross-sectional CT/ MRI imaging should be evident within 4 weeks after the start of steroid therapy (24). Some studies suggested that two weeks of steroid therapy might be sufficient to determine the response (i.e. resolution of abnormal imaging, and improvement in clinical and biochemical parameters), which may be of particular importance to avoid any delay in differentiating the AIP from pancreatic cancer $(27,28)$.

However, subjective improvement in symptoms or decline in serum IgG4 levels can occur even in pancreatic cancer and should not be used as separate response criteria (24).

Recently, US Mayo Clinic and Japan Pancreas Society outlined strategies to distinguish between AIP a pancreatic cancer $(15,28)$. 
According to the Mayo Clinic Strategy, CT findings should be used to stratify the patients into 3 groups: 1) highly suggestive of the AIP, 2) indeterminate (supportive of the AIP) and 3) highly suggestive of the pancreatic cancer. All the patients in groups 2 and 3 should undergo a workup for pancreatic cancer and the AIP; because pancreatic cancer is on balance of probabilities more likely than AIP, a workup for pancreatic cancer is the first step (biopsy of the lesion, CA 19-9 level in serum, and metastasis evaluation). In most (70 \%) patients, AIP is successfully diagnosed by pancreatic CT imaging, serum IgG4 levels, and determination of other organ involvement. But in patients without supportive serologic evidence or other organ involvement, the definitive diagnosis of AIP requires a pancreatic core biopsy, steroid trial, or operative intervention. Steroid trial (Prednison $40 \mathrm{mg}$ /day for two weeks) is strongly discouraged in the absence of collateral evidence for the AIP and in the positive workup for the cancer (15).

Japanese strategy differentiates AIP from pancreatic cancer in patients presenting with mass-like lesion in the pancreatic head. This strategy relies on radiologic cross-sectional imaging, serologic IgG4 level, and histologic data obtained by resection or biopsy. An endoscopic or percutaneous biliary drainage with cytologic examination of the biliary or pancreatic ducts is recommended routinely (28).

Japanese strategy concerns only mass-forming AIP, but Mayo Clinic strategy evaluates all imaging type of AIP. Both groups reported that the use of their strategies did not result in the inappropriate treatment of cancer with steroids (24). Strategies for distinguishing between AIP a pancreatic cancer have strengths and weaknesses, and they reflect differences in clinical practice in the USA and Japan.

\section{Conclusion}

It is generally accepted that an accurate diagnosis of the AIP can avoid major pancreatic surgery.

The role of the surgery in AIP remains still to be exactly determined.

But there are some arguments that support the importance of pancreatic surgery in AIP:

- The relapse rate in patients with type 1 AIP ranges from 30-50 \% $(4,12,29)$.

- Relapses are associated with chronic pancreatic injury (including development of pancreatic duct stones in 33-55\%) (16).

- More than $50 \%$ of patients with AIP may have findings, which are highly suggestive of pancreatic cancer (including elevated CEA, CA 19-9, and bile duct stenosis) (30).

- Case reports of pancreatic cancer associated with the AIP are described (31-34).

- Recent studies have highlighted an increased risk of malignancy (about 3-5 times greater than the general population) in patients with IgG4-related disease (35) and in patients with the AIP (36).

- Almost $15 \%$ of patients with AIP developed cancer, with the highest risk of occurring within the first year after the diagnosis of AIP (37).
- The patchy involvement of AIP limits histologic diagnosis (16).

- The consensus guidelines (International Consensus Diagnostic Criteria) for the AIP discourage the use of the diagnostic steroid trials alone to diagnose AIP. As IgG4 elevation associated with pancreatic cancer may decline and give a false diagnosis of autoimmune pancreatitis (6).

We believe, that surgical intervention is necessary as a first step in patient with pancreatic masses (despite suspicion on AIP) in the subgroup of patient with unclear diagnosis, with endoscopically untreated obstructive jaundice (because of risk of acute cholangitis), with probable coincidence of AIP and pancreatic cancer or in patients that refused pancreatic biopsy.

\section{References}

1. Yoshida K, Toki F, Takeuchi T, Watanabe S, Shiratori K, Hayashi N. Chronic pancreatitis caused by an autoimmune abnormality. Proposal of the concept of autoimmune pancreatitis. Dig Dis Sci 1995; 40 (7): 1 561-568.

2. Kanno A, Nishimori I, Masamune A, Kikuta K, Hirota M, Kuriyama $\mathrm{S}$ et al. Nationwide epidemiological survey of autoimmune pancreatitis in Japan. Pancreas 2012; 41 (6): 835-839.

3. Nishimori I, Tamakoshi A, Otsuki M. Prevalence of autoimmune pancreatitis in Japan from a nationwide survey in 2002. J Gastroenterol 2007; 42 (Suppl 18): 6-8.

4. Dite P, Nechutova H, Uvirova M, Dvorackova J, Kianicka B, Martinek A. Autoimmune pancreatitis. Biomed Papers Med Fac Uni Palacky Olomouc Czechoslov 2014; 158 (1): 17-22.

5. Chari ST, Kloeppel G, Zhang L, Notohara K, Lerch MM, Shimosegawa T. Histopathologic and Clinical Subtypes of Autoimmune Pancreatitis. Pancreas 2010; 39 (5): 549-554.

6. Shimosegawa T, Chari ST, Frulloni L, Kamisawa T, Kawa S, MinoKenudson $\mathrm{M}$ et al. International consensus diagnostic criteria for autoimmune pancreatitis: guidelines of the International Association of Pancreatology. Pancreas 2011; 40 (3): 352-358.

7. Umehara H, Okazaki K, Masaki Y, Kawano M, Yamamoto M, Saeki T et al. A novel clinical entity, IgG4-related disease (IgG4RD): general concept and details. Modern Rheum Jap Rheum Assoc 2012; 22 (1): 1-14.

8. Okazaki K, Uchida K, Ikeura T, Takaoka M. Current concept and diagnosis of IgG4-related disease in the hepato-bilio-pancreatic system. J Gastroenterol 2013; 48 (3): 303-314.

9. Ghazale A, Chari ST, Smyrk TC, Levy MJ, Topazian MD, Takahashi N et al. Value of serum IgG4 in the diagnosis of autoimmune pancreatitis and in distinguishing it from pancreatic cancer. Am J Gastroenterol 2007; 102 (8): 1646-1653.

10. Umehara H, Okazaki K, Masaki Y, Kawano M, Yamamoto M, Saeki T et al. Comprehensive diagnostic criteria for IgG4-related disease (IgG4-RD), 2011. Modern Rheum Jap Rheum Assoc 2012; 22 (1): 21-30.

11. Sah RP, Chari ST. Autoimmune pancreatitis: an update on classification, diagnosis, natural history and management. Curr Gastroenterol Rep 2012; 14 (2): 95-105.

12. Kamisawa T, Chari ST, Giday SA, Kim M, Chung JB, Lee KT et al. Clinical profile of autoimmune pancreatitis and its histological subtypes: an international multicenter survey. Pancreas 2011; 40 (6): 809-814. 
13. Chari ST, Smyrk TC, Levy MJ, Topazian MD, Takahashi N, Zhang L et al. Diagnosis of autoimmune pancreatitis: the Mayo Clinic experience. Clin Gastroenterol Hepatol 2006; 4 (8): 1010-1016.

14. Proctor RD, Rofe CJ, Bryant, T J C, Hacking CN, Stedman B. Autoimmune pancreatitis: an illustrated guide to diagnosis. Clin Radiol 2013; 68 (4): 422-432.

15. Chari ST, Takahashi N, Levy MJ, Smyrk TC, Clain JE, Pearson RK et al. A diagnostic strategy to distinguish autoimmune pancreatitis from pancreatic cancer. Clin Gastroenterol Hepatol 2009; 7 (10): 1097-1103.

16. Jani N, Buxbaum J. Autoimmune pancreatitis and cholangitis. World J Gastrointest Pharmacol Ther 2015; 6 (4): 199-206.

17. Iwashita T, Yasuda I, Doi S, Ando N, Nakashima M, Adachi S et al. Use of samples from endoscopic ultrasound-guided 19-gauge fine-needle aspiration in diagnosis of autoimmune pancreatitis. Clin Gastroenterol Hepatol 2012; 10 (3): 316-322.

18. Detlefsen S, Mortensen MB, Pless TK, Cribe A, de Muckadell, Ove B Schaffalitzky. Laparoscopic and Percutaneous Core Needle Biopsy Plays a Central Role for the Diagnosis of Autoimmune Pancreatitis in a Single-Center Study From Denmark. Pancreas 2015; 44 (6): 845-858.

19. Pannala R, Chari ST. Corticosteroid treatment for autoimmune pancreatitis. Gut 2009; 58 (11): 1438-1439.

20. Sandanayake NS, Church NI, Chapman MH, Johnson GJ, Dhar DK, Amin Z et al. Presentation and management of post-treatment relapse in autoimmune pancreatitis/immunoglobulin G4-associated cholangitis. Clin Gastroenterol Hepatol 2009; 7 (10): 1089-1096.

21. Kamisawa T, Shimosegawa T, Okazaki K, Nishino T, Watanabe H, Kanno A et al. Standard steroid treatment for autoimmune pancreatitis. Gut 2009; 58 (11): 1504-1507.

22. Dite P, Uvirova M, Bojkova M, Novotny I, Dvorackova J, Kianicka B et al. Differentiating autoimmune pancreatitis from pancreatic cancer. Minerva Gastroenterol Dietol 2014; 60 (4): 247-253.

23. Finkelberg DL, Sahani D, Deshpande V, Brugge WR. Autoimmune pancreatitis. N Engl J Med 2006; 355 (25): 2670-2676.

24. Agrawal S, Daruwala C, Khurana J. Distinguishing autoimmune pancreatitis from pancreaticobiliary cancers: current strategy. Anna Surg 2012; 255 (2): 248-258.

25. Sah RP, Chari ST. Serologic issues in IgG4-related systemic disease and autoimmune pancreatitis. Curr Opinion Rheumatol 2011; 23 (1): 108-113.
26. Bojkova M, Dite P, Dvorackova J, Novotny I, Floreanova K, Kianicka $\mathbf{B}$ et al. Immunoglobulin G4, autoimmune pancreatitis and pancreatic cancer. Digest Dis (Basel) 2015; 33 (1): 86-90.

27. Moon S, Kim M, Park DH, Hwang CY, Park SJ, Lee SS et al. Is a 2-week steroid trial after initial negative investigation for malignancy useful in differentiating autoimmune pancreatitis from pancreatic cancer? A prospective outcome study. Gut 2008; 57 (12): 1704-1712.

28. Kamisawa T, Imai M, Yui Chen P, Tu Y, Egawa N, Tsuruta K et al. Strategy for differentiating autoimmune pancreatitis from pancreatic cancer. Pancreas 2008; 37 (3): e62-67.

29. Sah RP, Chari ST, Pannala R, Sugumar A, Clain JE, Levy MJ et al. Differences in clinical profile and relapse rate of type 1 versus type 2 autoimmune pancreatitis. Gastroenterology 2010; 139 (1): 140-148.

30. Kamisawa T, Egawa $\mathbf{N}$, Nakajima $\mathbf{H}$. Autoimmune pancreatitis is a systemic autoimmune disease. Am J Gastroenterol 2003; 98 (12): 28112812.

31. Inoue H, Miyatani H, Sawada Y, Yoshida Y. A case of pancreas cancer with autoimmune pancreatitis. Pancreas 2006; 33 (2): 208-209.

32. Fukui T, Mitsuyama T, Takaoka M, Uchida K, Matsushita M, Okazaki K. Pancreatic cancer associated with autoimmune pancreatitis in remission. Intern Med (Japan) 2008; 47 (3): 151-155.

33. Urata T, Naito Y, Izumi Y, Takekuma Y, Yokomizo H, Nagamine $\mathbf{M}$ et al. Localized type 1 autoimmune pancreatitis superimposed upon preexisting intraductal papillary mucinous neoplasms. World J Gastroenterol 2013; 19 (47): 9127-9132.

34. Ikeura T, Miyoshi H, Uchida K, Fukui T, Shimatani M, Fukui Y et al. Relationship between autoimmune pancreatitis and pancreatic cancer: a single-center experience. Pancreatology 2014; 14 (5): 373-379.

35. Yamamoto M, Takahashi H, Tabeya T, Suzuki C, Naishiro Y, Ishigami K et al. Risk of malignancies in IgG4-related disease. Modern Rheum Jap Rheum Assoc 2012; 22 (3): 414-418.

36. Hart PA, Kamisawa T, Brugge WR, Chung JB, Culver EL, Czako L et al. Long-term outcomes of autoimmune pancreatitis: a multicentre, international analysis. Gut 2013; 62 (12): 1771-1776.

37. Shiokawa M, Kodama Y, Yoshimura K, Kawanami C, Mimura J, Yamashita $\mathbf{Y}$ et al. Risk of cancer in patients with autoimmune pancreatitis. Am J Gastroenterol 2013; 108 (4): 610-617.

Received November 30, 2015. Accepted February 5, 2016. 